\title{
Actin regulation during abscission: unexpected roles of Rab35 and endocytic transport
}

\author{
Rytis Prekeris $^{1}$ \\ ${ }^{I}$ Department of Cell and Developmental Biology, School of Medicine, University of Colorado, 12801 E. $17^{\text {th }}$ Avenue, Aurora, CO \\ 80045, USA \\ Cell Research (2011) 21:1283-1285. doi:10.1038/cr.2011.131; published online 16 August 2011
}

The final abscission event of cytokinesis is a complex cellular event that results in the separation of daughter cells and the completion of cell division. Multiple studies during the last decade have identified numerous proteins that have been shown to be required for the abscission, although the mechanisms and regulation of the abscission event are only beginning to emerge. It has become clear that the actin cytoskeleton plays a pivotal role in the initiation and completion of cytokinesis (reviewed in [1]). Indeed, cytokinesis starts during anaphase as a result of the assembly and contraction of the acto-myosin ring, which drives the formation and ingression of the cleavage furrow. The machinery regulating the formation of the acto-myosin ring is well established and is known to involve mitotic spindle microtubules as well as several specialized signaling complexes, such as the centralspindlin and chromosomal passenger complex (CPC). Centralspindlin and CPC lead to the activation of small monomeric GTPase RhoA, eventually resulting in the stabilization and contraction of the acto-myosin ring. Interestingly, while formation of actin cytoskeletal network is required during early cytokinesis, actin depolymer-

Correspondence: Rytis Prekeris

E-mail: Rytis.Prekeris@ucdenver.edu ization within the cleavage furrow is needed for abscission, presumably to allow the thinning of the intracellular bridge (ICB) connecting daughter cells. The removal of the actin cytoskeleton also would be required for the fusion of the opposing plasma membranes within the ICB, which directly leads to the abscission event.

In addition to RhoA GTPase, phosphoinositides also have emerged as important regulators of the actin cytoskeleton during cytokinesis. Phosphatidylinositol-4,5-bisphosphate (PtdIns $\left.(4,5) \mathrm{P}_{2}\right)$ is a well-established regulator of the actin cytoskeleton during interphase. Recent work also has shown that PtdIns $(4,5) \mathrm{P}_{2}$ accumulates at the cleavage furrow in yeast, $\mathrm{Dic}$ tyostelium and mammalian cells $[2,3]$. Furthermore, inhibition of $\operatorname{PtdIns}(4,5)$ $\mathrm{P}_{2}$ production leads to the failure of cytokinesis [2-4]. While the roles of PtdIns $(4,5) \mathrm{P}_{2}$ during cytokinesis remains to be fully elucidated, at least some of its functions are known: the stabilization of the acto-myosin contractile ring, as well as the linking of the actin cytoskeleton to the plasma membrane by the virtue of its binding to septins and ERM (exrin/ radixin/moesin) proteins [5]. Consistent with that, the levels of $\operatorname{PtdIns}(4,5) \mathrm{P}_{2}$ within the ICB decreases as the actin cytoskeleton is disassembled before cells progress to abscission [6]. Since PtdIns $(4,5) \mathrm{P}_{2}$ accumulation in the fur- row is reminiscent of the polarization of $\operatorname{PtdIns}(4,5) \mathrm{P}_{2}$ during cell motility, it has been proposed that proteins such as phophatidylinositol-4-phosphate 5-kinase (PI5K $\beta$ ) and phosphatase and tensin homologue on chromosome 10 (PTEN) may also regulate PtdIns $(4,5) \mathrm{P}_{2}$ accumulation at the ingressing furrow. Indeed, PI5K $\beta$ and PTEN were shown to be enriched during furrow formation and be required for early cytokinesis $[3,4]$. What is much less understood is how the levels of $\operatorname{PtdIns}(4,5) \mathrm{P}_{2}$ are downregulated during abscission. In a recent paper published in Nature Cell Biology, Echard and colleagues propose a novel and somewhat unexpected mechanism of regulating the levels of PtdIns $(4,5) \mathrm{P}_{2}$ within the ICB during late telophase [7]. This study is based on earlier findings that the small monomeric GTPase Rab35 regulates the levels of PtdIns $(4,5) \mathrm{P}_{2}$ within the cleavage furrow during anaphase and early telophase [8]. Indeed, overexpression of the Rab35 dominant-negative mutant (Rab35-S22N) resulted in the dramatic reduction of PtdIns(4,5) $\mathrm{P}_{2}$ within the ingressing furrow and accumulation of PtdIns $(4,5) \mathrm{P}_{2}$ in the enlarged endocytic organelles [8]. Furthermore, Rab35S22N also inhibited recruitment of septin 2 and the actin cytoskeleton to the forming furrow, demonstrating that Rab35 is required for PtdIns(4,5) $\mathrm{P}_{2}$ and actin filament accumulation within the 
cleavage furrow during early telophase [8].

All Rab GTPases cycle between GDP-bound (inactive) and GTP-bound (active) forms. Rabs function by binding various effector proteins and recruiting them to membranes. To understand the mechanisms of Rab35's function, Echard set out to identify its binding effector proteins by completing yeast two hybrid screen [7]. One of the proteins isolated in this screen turned out to be phosphatidylinositol-4,5-bisphosphate 5-phosphatase, OCRL [7]. Consistent with OCRL being a Rab35 effector protein, it was shown to bind to GTP-Rab35 but not GDP-Rab35 via immunoprecipitation, glutathione bead pull-down and solid-phase assays [8]. Finally, using Rab35 knockdown assays, the authors have demonstrated that Rab35 recruits OCRL specifically to the ICB during late telophase. Surprisingly, OCRL functions as PtdIns $(4,5) \mathrm{P}_{2}$ phosphatase, thus mediating the decrease rather than the accumulation of $\operatorname{PtdIns}(4,5) \mathrm{P}_{2}$ within the furrow. Consistent with these data, the authors have demonstrated that OCRL functions during late telophase to mediate actin cytoskeleton disassembly, presumably by inactivating PtdIns $(4,5) \mathrm{P}_{2}$, since OCRL knockdown led to the accumulation of $\operatorname{PtdIns}(4,5)$ $\mathrm{P}_{2}$ and actin within the ICB during late telophase and subsequent delay of the abscission step of cytokinesis [7]. Furthermore, OCRL knockdown could be only rescued with wild-type but not phosphatase-dead GFP-OCRL (OCRL-H524R). The discovery that OCRL is a Rab35 effector protein seems to disagree with the author's original conclusion that Rab35 mediates the accumulation of PtdIns $(4,5) \mathrm{P}_{2}$ within the forming ingression furrow [8]. These controversial conclusions turned out to have a very intriguing explanation: Rab35 has distinct and opposing functions during anaphase/early telophase and abscission. Echard and colleagues propose that during anaphase and early telophase Rab35 increases the levels of
$\operatorname{PtdIns}(4,5) \mathrm{P}_{2}$ at the ingressing cleavage furrow, thus inducing the accumulation of the furrow actin cytoskeleton [7]. Although the exact mechanism of Rab35's action during early cytokinesis remains unclear, it is likely that it acts along-side other known mitotic actin regulators, such as the Centralspindlin complex and RhoA GTPase. In contrast, during late telophase Rab35 binds to and recruits OCRL to the ICB, where OCRL dephosphorylates PtdIns(4,5) $\mathrm{P}_{2}$, thus leading to localized actin disassembly within the future abscission site. This biphasic regulation of actin and PtdIns(4,5)P $\mathrm{P}_{2}$ by Rab35 GTPase still needs to be conclusively demonstrated. However, this study's observation that high overexpression of the Rab35 dominant-negative mutant blocks cytokinesis at early telophase, while low overexpression of the same mutant allows cells to proceed to late telophase and only delays the abscission step, is consistent with this hypothesis. Similarly, partial knockdown of Rab35 also allows cells to complete furrow formation and ingression while blocking PtdIns(4,5) $\mathrm{P}_{2}$ and actin cytoskeleton disassmebly within the ICB, a phenotype reminiscent of OCRL knockdown.

While this study clearly demonstrates that Rab35 and OCRL are directly involved in regulating the levels of $\operatorname{PtdIns}(4,5) \mathrm{P}_{2}$ and actin within the ICB, many questions remain. What is the Rab35 effector protein(s) during anaphase/early telophase? How does Rab35 switch to binding to OCRL during late telophase? Finally, is Rab35 the sole regulator of actin disassembly during late telophase or does it act in concert with RhoA inactivation? These questions remain to be answered and will be the focus of future investigations by many laboratories.

In addition to demonstrating the complexity of actin and PtdIns(4,5) $\mathrm{P}_{2}$ regulation by Rab35 and OCRL, this study has an interesting twist. OCRL has been originally described as a protein mutated in patients with oculocerebrorenal syndrome of Lowe [9]. Lowe syndrome is a multisystem disorder affecting the kidneys, eyes and nervous system. While Lowe syndrome symptoms are likely caused by multiple cellular abnormalities, such as defects in signaling, endocytic transport, the maintenance and function of tight and adherens junctions and protein trafficking, this work suggests that at least some of the pathologies may be a result of abnormal cell division due to the accumulation of the actin cytoskeleton within the ICB. Consistent with this, treatment of isolated Lowe patient renal cells with low levels $(1-4 \mathrm{nM})$ of the actin depolymerizing drug latrunculin-A almost completely rescued the cytokinesis phenotype, while having no visible effect on wild-type cells [7]. This raises an interesting possibility that at least some of the Lowe syndrome pathologies may be treated with actin depolymerizing drugs, thus providing potential new therapeutic avenues for the treatment of this disorder.

\section{References}

1 Pollard TD. Mechanics of cytokinesis in eukaryotes. Curr Opin Cell Biol 2010; 22:50-56.

2 Emoto K, Umeda M. An essential role for a membrane lipid in cytokinesis. Regulation of contractile ring disassembly by redistribution of phosphatidylethanolamine. J Cell Biol 2000; 149:1215-1224.

3 Field SJ, Madson N, Kerr ML, et al. PtdIns(4,5)P2 functions at the cleavage furrow during cytokinesis. Curr Biol 2005; 15:1407-1412.

4 Janetopoulos C, Borleis J, Vazquez F, Iijima M, Devreotes P. Temporal and spatial regulation of phosphoinositide signaling mediates cytokinesis. Dev Cell 2005; 8:467-477.

5 Janetopoulos C, Devreotes P. Phosphoinositide signaling plays a key role in cytokinesis. J Cell Biol 2006; 174:485-490.

6 Wong R, Hadjiyanni I, Wei HC, et al. PIP2 hydrolysis and calcium release are required for cytokinesis in Drosophila spermatocytes. Curr Biol 
2005; 15:1401-1406.

7 Dambournet D, Machicoane M, Chesneau L, et al. Rab35 GTPase and OCRL phosphatase remodel lipids and F-actin for successful cytokinesis. Nat Cell Biol 2011; 13:981-988.
8 Kouranti I, Sachse M, Arouche N, Goud B, Echard A. Rab35 regulates an endocytic recycling pathway essential for the terminal steps of cytokinesis. Curr Biol 2006; 16:1719-1725.

9 Lowe CU, Terrey M, Mac LE. Organic- aciduria, decreased renal ammonia production, hydrophthalmos, and mental retardation; a clinical entity. AMA Am J Dis Child 1952; 83:164184. 